\title{
On the Formation Mechanisms of Hydrogen Ionic Clusters
}

\author{
M. Barbatti and M. A. C. Nascimento \\ Instituto de Química, Universidade Federal do Rio de Janeiro, \\ Rio de Janeiro, 21949-900, RJ, Brazil
}

Received on 13 March, 2003

\begin{abstract}
Structural and thermodynamic properties of hydrogen molecular clusters formed around an atomic or molecular cation are examined. The shell distribution of $\mathrm{H}_{2}$ molecules and the size of the clusters are discussed. The Bloom-Margenau model for predicting the number of neutral molecules that could bind to a cation core is investigated and its limitations are illustrated using the $\mathrm{Li}^{+}\left(\mathrm{H}_{2}\right)_{k}$ clusters as test case. Finally, results for the entropy of the $\mathrm{H}_{n}^{+}$clusters $(n=5-27$, odd) and for the Gibbs free energy variations associated to the cluster formation are presented and the spontaneity of the clustering process in different conditions is examined.
\end{abstract}

\section{Introduction}

The presence of a cation in a molecular hydrogen environment leads to the formation of molecular clusters around the ion. In the case of an $\mathrm{H}_{2}$ homogeneous atmosphere, an $\mathrm{H}_{2}^{+}$ ion is quickly converted to the $\mathrm{H}_{3}^{+}$molecule, which becomes the core for the clustering process

$$
\mathrm{H}_{3}^{+}+(k+1) \mathrm{H}_{2} \rightarrow \mathrm{H}_{3}^{+}\left(\mathrm{H}_{2}\right)_{k}+\mathrm{H}_{2},
$$

where the exceeding $\mathrm{H}_{2}$ molecule carries away the excess of energy, stabilizing the cluster. From these multiple-steps reactions, $\mathrm{H}_{n}^{+}$clusters ( $n$ odd) are formed, and clusters as large as $\mathrm{H}_{99}^{+}$have been observed [1]. Depending on the temperature and pressure conditions, the $\mathrm{H}_{15}^{+}$and $\mathrm{H}_{17}^{+}$clusters are the more abundant.

One of the most interesting features of these species is that in the cluster, each $\mathrm{H}_{2}$ molecule is strongly bound by the coulombian field of the cation. This may be very useful when dealing with hydrogen storage problems.

In the last decade, $a b$ initio calculations have been performed to clusters as large as $\mathrm{H}_{35}^{+}$[2-12]. Recently, more attention has been dedicated to the $\mathrm{H}_{5}^{+}$cluster. Its potential energy surface has been studied at high levels of calculation $[13,14]$ and its presence in some interstellar environments in concentrations up two times higher than that of the $\mathrm{H}_{3}^{+}$ molecule has been investigated [15].

Besides the $\mathrm{H}_{3}^{+}$molecular ion, a large variety of atomic and molecular cations has been considered as a core for $\mathrm{H}_{2}$ clustering: the first-column $\mathrm{Li}^{+}, \mathrm{Na}^{+}$and $\mathrm{K}^{+}$ions [16-22]; the second-column $\mathrm{Be}^{+}$and $\mathrm{Mg}^{+}$ions [23]; the $\mathrm{Al}^{+}$ion and all metals from $\mathrm{Sc}^{+}$to the $\mathrm{Zn}^{+}$ions [24, 25, 26]; and the molecular ions $\mathrm{LiH}^{+}$[21], $\mathrm{Li}_{3}^{+}$[27], $\mathrm{BeH}^{+}$[28], $\mathrm{CH}_{5}^{+}$ $[29,30], \mathrm{N}_{2} \mathrm{H}^{+}$[31] and $\mathrm{CH}_{3} \mathrm{~N}_{2}^{+}$[32]. Table I summarizes some properties of these $\mathrm{X}^{+}\left(\mathrm{H}_{2}\right)_{k}$ hydrogen clusters.

The experimental work on the hydrogen clusters has been concentrated on enthalpy variation measurements
$(\Delta H)[16,24,25,26,31,33]$ and collisional induced dissociation (CID) studies $[34,35,36,37]$. The $\Delta H$ measurements for the $\mathrm{H}_{n}^{+}$clusters and for the most general $\mathrm{X}^{+}\left(\mathrm{H}_{2}\right)_{k}$ clusters have been an important source of information about the cluster energetic properties and have guided the theoretical studies. On the other hand, the CID studies have revealed patterns for the cluster dissociation and their dependence on the shell structure of $\mathrm{H}_{2}$ distribution. Hitherto, very few theoretical and experimental attention has been payed to the vibrational properties and the infrared spectra of the hydrogen clusters, these studies being basically restricted to the $\mathrm{H}_{5}^{+}$species [38, 39]. For clusters larger than the $\mathrm{H}_{5}^{+}$one, only a few experimental results are available [42] and the theoretical calculations have been limited to the harmonics frequencies [14, 40, 41].

In the next section we discuss the structure of the clusters and the question of the shell distribution of the $\mathrm{H}_{2}$ molecules. Following that, we discuss the size of the cluster, based on a classical thermodynamical model. Finally, we discuss the relative stability of these clusters based on the results for the entropy and Gibbs free energy of the clusters.

\section{The structure of the hydrogen clus- ters}

The hydrogen clusters are formed around an atomic or molecular cation $\mathrm{X}^{+}$by means of exothermic multiple-step reactions of the type

$$
\mathrm{X}^{+}+(k+1) \mathrm{H}_{2} \rightarrow \mathrm{X}^{+}\left(\mathrm{H}_{2}\right)_{k}+\mathrm{H}_{2} .
$$

The $\mathrm{H}_{2}$ molecules are bound to the cation mainly by monopole/induced-dipole interactions, although some charge transfer from the nearest $\mathrm{H}_{2}$ units to the cation is also observed. 
Table I. Maximum occupation number $\left(\mathrm{H}_{2}\right.$ units) of the successive shells around the core $\mathrm{X}^{+}$. Binding energy (and enthalpy) of the first $\mathrm{H}_{2}$ molecule bound to the core.

\begin{tabular}{lll}
\hline $\mathrm{X}^{+}$ & $\mathrm{H}_{2}$ number & $-D_{e}\left[-\Delta H^{0}\right](\mathrm{kcal} / \mathrm{mol})$ \\
\hline $\mathrm{H}_{3}^{+}$ & $3,6,(13 \pm 2)^{g}$ & $7,9^{a}$ \\
$\mathrm{Li}^{+}$ & 6 & $5,9^{b}$ \\
$\mathrm{Li}_{3}^{+}$ & 12 & $3,1^{c}$ \\
$\mathrm{CH}_{5}^{+}$ & 5 & $3,5^{d}$ \\
$\mathrm{CH}_{3} \mathrm{~N}_{2}^{+}$ & $3,3,3$ & $0,9^{e}$ \\
$\mathrm{~N}_{2} \mathrm{H}^{+}$ & $1, \geq 6$ & {$[6,0]^{f}$} \\
$\mathrm{Na}^{+}$ & $>7$ & $3,0^{b}$ \\
$\mathrm{~K}^{+}$ & $(10)^{g}$ & $1,2^{b}$ \\
$\mathrm{~V}^{+}$ & - & {$[10,2]^{h}$} \\
$\mathrm{Ti}^{+}, \mathrm{Cr}^{+}, \mathrm{Fe}^{+}, \mathrm{Co}^{+}$ & 6 & {$[10,0],[7,6],[16,5],[18,2]^{h}$} \\
$\mathrm{Ni}^{+}$ & 5 & {$[17,3]^{h}$} \\
$\mathrm{Mn}^{+}$ & $>6$ & $3,4^{h}$ \\
$\mathrm{Cu}^{+}$ & 4 & $18,6^{h}$ \\
$\mathrm{Zn}^{+}$ & - & $6,1^{h}$ \\
\hline
\end{tabular}

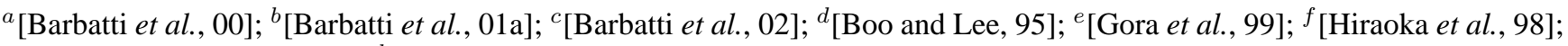
${ }^{g}$ extrapolated (present work); ${ }^{h}$ [Weis et al., 97 and Kemper et al., 98].

The binding energy of the $\mathrm{H}_{2}$ molecules is in the range of 0.5 and $20.0 \mathrm{kcal} / \mathrm{mol}$ depending on the distance from the $\mathrm{H}_{2}$ unit to the core and the orbital features of the core. These binding energies are small if compared to the $\mathrm{H}_{2}$ internal binding energy (about $100 \mathrm{kcal} / \mathrm{mol}$ in the $\mathrm{H}+\mathrm{H}$ channel), implying that the $\mathrm{H}_{2}$ units keep their molecular identities within the cluster. This same reasoning applied to the $\mathrm{H}_{3}^{+}$ core molecule explains why the clustering occurs around a well defined $\mathrm{H}_{3}^{+}$molecule.

The results of high-level ab initio calculations [11] revealed that the $\mathrm{H}_{2}$ molecules are distributed in shells around the core (see Fig. 1), and they allowed the characterization of a shell as a set of $\mathrm{H}_{2}$ molecules sharing similar geometrical and energetic properties. The number of $\mathrm{H}_{2}$ molecules in each shell as well as other features of the shells depend on several factors: (i) the coulombian field of the core, which defines a radial pattern of distribution; (ii) the geometrical and the orbital structures of the core, both determining the symmetry of the distribution of ligands and the first-shell features; (iii) the $\mathrm{H}_{2}-\mathrm{H}_{2}$ interactions, which contribute to define the number of $\mathrm{H}_{2}$ molecules in each shell.
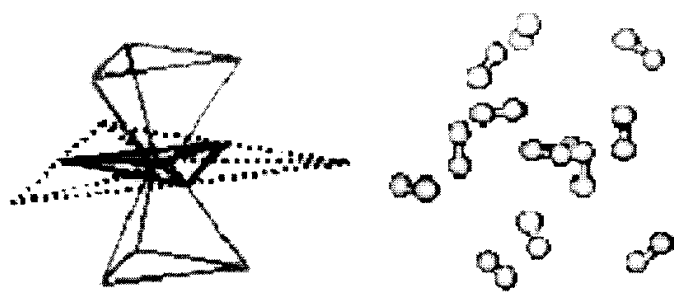

Figure 1. Geometry of $\mathrm{H}_{27}^{+}$cluster at its equilibrium configuration. On the left, the several kinds of lines indicates the different shells in which the $\mathrm{H}_{2}$ units are distributed.

It is remarkable that the number of $\mathrm{H}_{2}$ molecules in each shell is such that the $\mathrm{H}_{2}-\mathrm{H}_{2}$ distance within a shell resembles that one of the first-neighbors in solid hydrogen $(3.8 \AA)$.
This implies the existence of long-distance collective effects acting upon the cluster structure. Note, in particular, that the van der Waals energy minimum for the $\mathrm{H}_{2}-\mathrm{H}_{2}$ interaction occurs at $4.53 \AA$. By suposing that in the third shell the intermolecular distance is $3.8 \AA$, we may estimate that the number of $\mathrm{H}_{2}$ units in this shell is around thirteen (Table ).

Although the $\mathrm{X}^{+}$core and the $\mathrm{H}_{2}$ units keep their molecular identities within the cluster, they are somehow perturbed by the environment. This can be clearly inferred from the observation that the $\mathrm{H}_{2}$ properties, such as equilibrium distance and vibrational frequencies, deviate from their values in the isolated $\mathrm{H}_{2}$. Of course, the deviations are as more significant as closer to core the $\mathrm{H}_{2}$ units are. Assymptoticaly, as $k$ becomes large, these properties tend to a value still slightly different from the isolated $\mathrm{H}_{2}$, reflecting the environment influence. This effect is illustrated in Fig. 2a for the case of the vibrational frequencies of the $\mathrm{H}_{2}$ units. As expected, the $\mathrm{X}^{+}$core properties tend to a solvated value as $k$ becomes large, as shown in Fig. 2b. The calculational details are described in Section IV.

\section{On the number of molecules in the clusters}

An interesting problem related to the hydrogen ionic clusters is the determination of the number of $\mathrm{H}_{2}$ molecules which can be bound to an ionic core. The first theoretical model to calculate the number of neutral molecules which could bind to a cation was proposed by Bloom and Margenau [17]. In this section, this model is examined and its limitation are illustrated using the $\mathrm{Li}^{+}\left(\mathrm{H}_{2}\right)_{k}$ cluster as a test case.

Bloom and Margenau started by assuming a positively charged ion surrounded by $N$ neutral molecules with mass $m$. This system is supposed to have a very large radius $R$ and a constant temperature $T$. If the interaction potential 
between the cation and a molecule at the distance $r$ from it is given by $V(r)$, then the number $d N$ of molecules between $r$ and $r+d r$ and with velocity between $v$ and $v+d v$, according to the Maxwell-Boltzmann distribution, is given by

$$
d N=16 \rho \pi^{1 / 2}\left(\frac{m}{2 k_{B} T}\right)^{3 / 2} \exp \left[-\frac{\frac{1}{2} m v^{2}+V(r)}{k_{B} T}\right] r^{2} v^{2} d r d v,
$$

where $\rho=N /\left(\frac{4}{3} \pi R^{3}\right)$.

The number of molecules bound to the ion may be carried out by integration of Eq. 3, under the restriction

$$
\frac{1}{2} m v^{2}+V(r) \leq 0 .
$$

For each $r$, the largest velocity $v_{m}(r)$ achieved by a molecule satisfying the Eq. 4 is

$$
v_{m}(r)=\left(-\frac{2 V(r)}{m}\right)^{1 / 2}
$$

Therefore, the number $N_{b}$ of the molecules bound to the ion is

$$
N_{b}=16 \rho \pi^{1 / 2}\left(\frac{m}{2 k_{B} T}\right)^{3 / 2} \int_{r_{e}}^{\infty} \exp \left[-\frac{V(r)}{k_{B} T}\right] \phi(r) r^{2} d r
$$

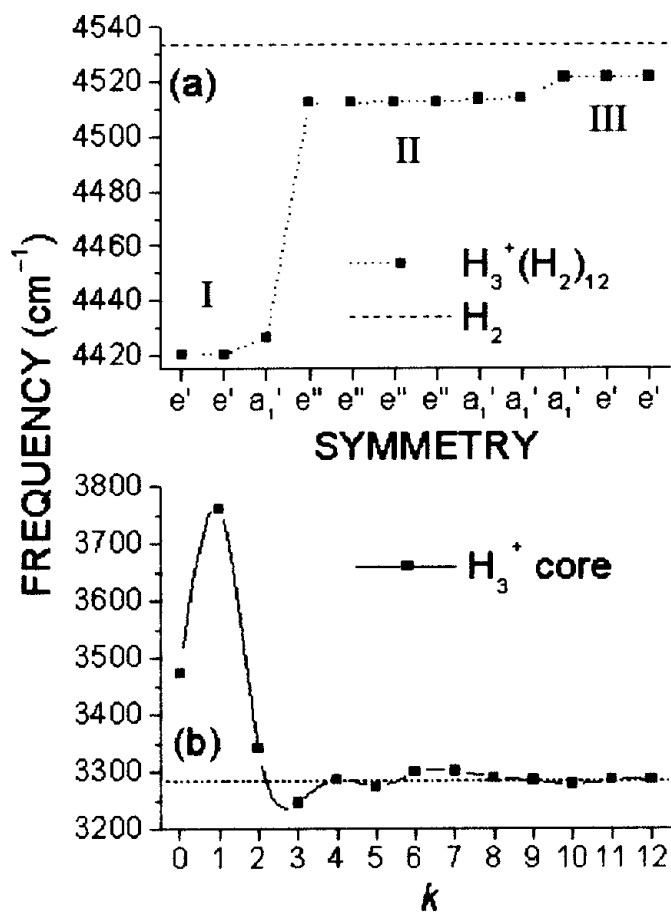

Figure 2. (a) Vibrational stretching harmonic frequencies of the $\mathrm{H}_{2}$ units in the $\mathrm{H}_{27}^{+}$cluster $(k=12)$. Although these frequencies correspond to collective vibrational modes, each one is composed just by $\mathrm{H}_{2}$ internal vibrations. At each level I, II, or III only the $\mathrm{H}_{2}$ units belonging to the shell I, II, or III are vibrating. Dashed line corresponds to the isolated $\mathrm{H}_{2}$ harmonic frequency. (b) $\mathrm{Vi}$ brational harmonic frequencies of the breathing mode of the $\mathrm{H}_{3}^{+}$ core within the cluster. Dashed line corresponds to the isolated $\mathrm{H}_{3}^{+}$ harmonic frequency. All frequencies were calculated at MP2/6-
$311 \mathrm{G}(\mathrm{p})$ level.

with

$$
\phi(r)=\int_{0}^{v_{m}(r)} \exp \left[-\frac{\frac{1}{2} m v^{2}}{k_{B} T}\right] v^{2} d v,
$$

where $r_{e}$ is the minimum distance between the ion and the molecule for which the interaction energy is negative (the classical return point).

To calculate $N_{b}$ one needs $V(r)$. Bloom and Margenau used a phenomenological potential that combines a shortdistance repulsive term $\left(r^{-12}\right)$ and a large-distance attractive monopole/induced-dipole term $\left(r^{-4}\right)$ :

$$
V(r)=\frac{1}{2} \alpha\left[r_{e}^{8} r^{-12}-r^{-4}\right],
$$

where $\alpha=0.806$ hartree- $\AA^{4}$ is the force constant of the $\mathrm{H}_{2}$ molecule. The integration of Eq. 6 can be performed numerically, and the result of Bloom and Margenau for the clustering of $\mathrm{H}_{2}$ molecules around $\mathrm{Li}^{+}$cation is shown in Fig. 4, for a minimum distance of approximation of $r_{e}=2.16 \AA$. The result is strongly dependent on the temperature, changing from almost one hundred of molecules bound to the core to just one, in the range from 100 to 200 $\mathrm{K}\left(\rho=2.687 \times 10^{19} \mathrm{~cm}^{-3}\right)$.

The results of Bloom-Margenau for the $\mathrm{Li}^{+}\left(\mathrm{H}_{2}\right)_{k}$ clusters suffer the inadequacy of the potential curve. The values for the parameters in Eq. 8 underestimate the binding energy $D_{e}$ and overestimate the equilibrium distance (Fig. 3). By using a more accurate $\mathrm{Li}^{+}-\mathrm{H}_{2}$ potential obtained from $a b$ initio calculations, performed at the same level as employed in Ref. [22], the result for the number of bound molecules 
changes drastically in comparison to those of Bloom and Margenau, as shown in the Fig. 4. With the more accurate potential, the clustering process becomes more efficient at higher temperatures, particularly in the range of temperatures for which the cluster formation is enthalpically more favored (around $190 \mathrm{~K}$ [11]).

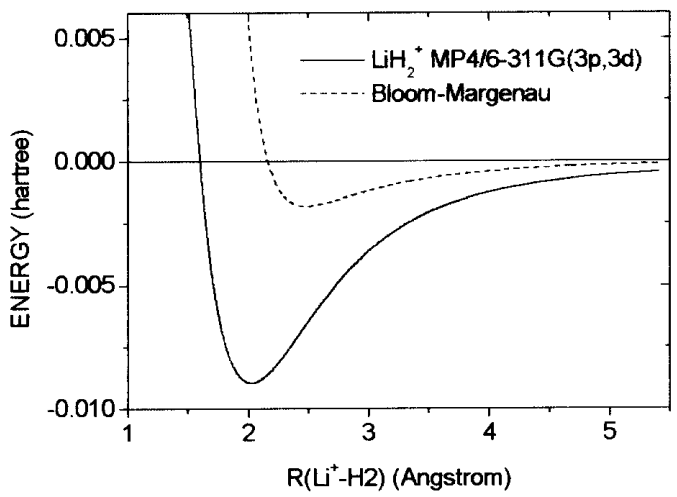

Figure 3. Potential energy along the $\mathrm{Li}^{+}-\mathrm{H}_{2}$ distance according to the Bloom-Margenau model (dashed line) and according to the present $a b$ initio calculation.

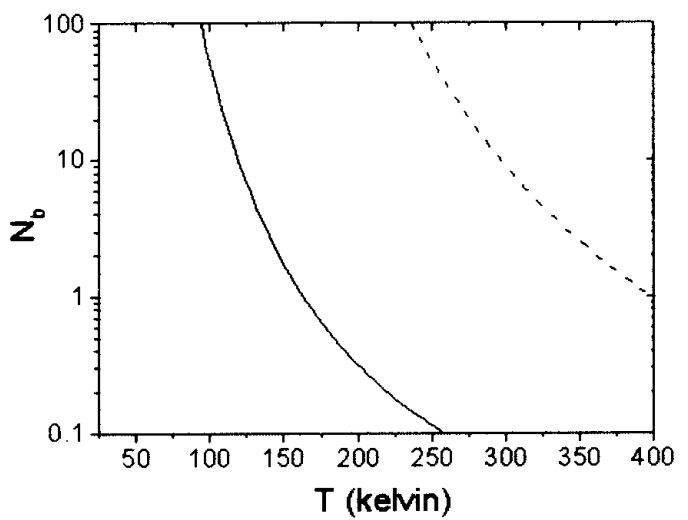

Figure 4. Number of $\mathrm{H}_{2}$ molecules bound to the $\mathrm{Li}^{+}$core according to the Bloom-Margenau model $\left(\rho=2.9 \times 10^{19} \mathrm{~cm}^{-3}\right)$. Solid line is the original result from Bloom and Margenau. Dashed line is the result obtained with $a b$ initio potential (see text).

Although the qualitative predictions from the BloomMargenau model may be improved by the use of more accurate potentials, the model still suffers from a major problem. By neglecting the $\mathrm{H}_{2}-\mathrm{H}_{2}$ interactions, which play the double role of stabilizing the cluster and also defining the number of molecules in a shell, the Bloom-Margenau model predicts that an infinite number of $\mathrm{H}_{2}$ units can bind to the cluster at lower temperatures.

\section{The clustering process in low- density atmospheres}

Another very interesting problem related to these clusters has to do with the spontaneity of the clustering process. It would be particularly interesting to examine the spontaneity of these processes in the same conditions where experiments have been conducted and also in conditions typical from dense interstellar clouds (DIC).

Elsewhere we have discussed the binding energy of several kinds of clusters and also the enthalpy variations $(\Delta H)$ for the clustering process $[11,27]$. The $\Delta H$ values could be combined with the entropy variations $(\Delta S)$ to obtain the Gibbs free energy changes $(\Delta G)$ for the clustering processes:

$$
\mathrm{H}_{n-2}^{+}+\mathrm{H}_{2} \rightleftharpoons \mathrm{H}_{n}^{+} \text {. }
$$

The entropy variation for the processes indicated in Eq.9 is given by:

$$
\Delta S=S\left(\mathrm{H}_{n}^{+}\right)-\left[S\left(\mathrm{H}_{n-2}^{+}\right)+S\left(\mathrm{H}_{2}\right)\right],
$$

The calculations follow very closely the ones described in ref. $[10,11]$, and only a brief discussion will be presented. The electronic energies were computed at the complete fourth-order perturbation theory (MP4) with 6-311G(2p) basis set and using Hartree-Fock wave functions as zero-order. In our previous studies, coupled cluster $\operatorname{CCSD}(\mathrm{T})$ calculations have been also considered to show the convergence of the MP4 results. Basis set superposition error (BSSE), computed according to counterpoise method of Boys and Bernardi [43], were always less than $0.03 \mathrm{kcal} / \mathrm{mol}$. The harmonic frequencies were calculated at the MP2 level and scaled by the factor 0.9223 to take anharmonic effects into account. The partition function were computed assuming ideal behavior for all the species indicated in Eq. 9. For the ionic species this approximation may be justified by the fact that we have restricted our analysis to systems at very low densities.

The entropy $(\Delta S)$ and the Gibbs free energy $(\Delta G)$ variations were computed for the following two cases: a) the conditions used in the magnetic trap experiments of ref.[44], that is a density of $10^{14} \mathrm{H}_{2}$ molecules $/ \mathrm{cm}^{3}\left(3 \times 10^{-16} \mathrm{~atm}\right)$ and a temperature of $10 \mathrm{~K}$; b) the typical conditions found at DICs: $\mathrm{H}_{2}$ density of $10^{5}$ molecules $/ \mathrm{cm}^{3}\left(2 \times 10^{-15} \mathrm{~atm}\right)$ and a temperature of $30 \mathrm{~K}$ [45].

Figure 5a shows the entropy for the clusters with $n=$ $5-27$. From that figure, it is clear that the entropy increases monotonically with the cluster size, exhibiting an almost logarithmic behavior, with slight deviations for the $\mathrm{H}_{9}^{+}, \mathrm{H}_{15}^{+}$and $\mathrm{H}_{21}^{+}$clusters. These deviations can be understood in terms of the different contributions to the entropy. By comparing the translational, vibrational and rotational entropy contributions for the $\mathrm{H}_{19}^{+}, \mathrm{H}_{21}^{+}$and $\mathrm{H}_{23}^{+}$clusters, we see that the reduction of the entropy for the $\mathrm{H}_{21}^{+}$cluster is due to a decrease in the vibrational and rotational contributions (Fig. 6). The same effects are responsible for the decrease in the entropy for the $\mathrm{H}_{9}^{+}$and $\mathrm{H}_{15}^{+}$clusters.

The decrease in the rotational contributions is a consequence of the great number of indistinguishable orientations allowed by the highly symmetric equilibrium geometry of these clusters. In the case of the $\mathrm{H}_{27}^{+}$cluster, that also presents a highly symmetric structure, the minimum of entropy is not observed because the $\mathrm{H}_{27}^{+}$moment of inertia is much higher than that of the $\mathrm{H}_{25}^{+}$cluster, leading to a compensatory effect. 

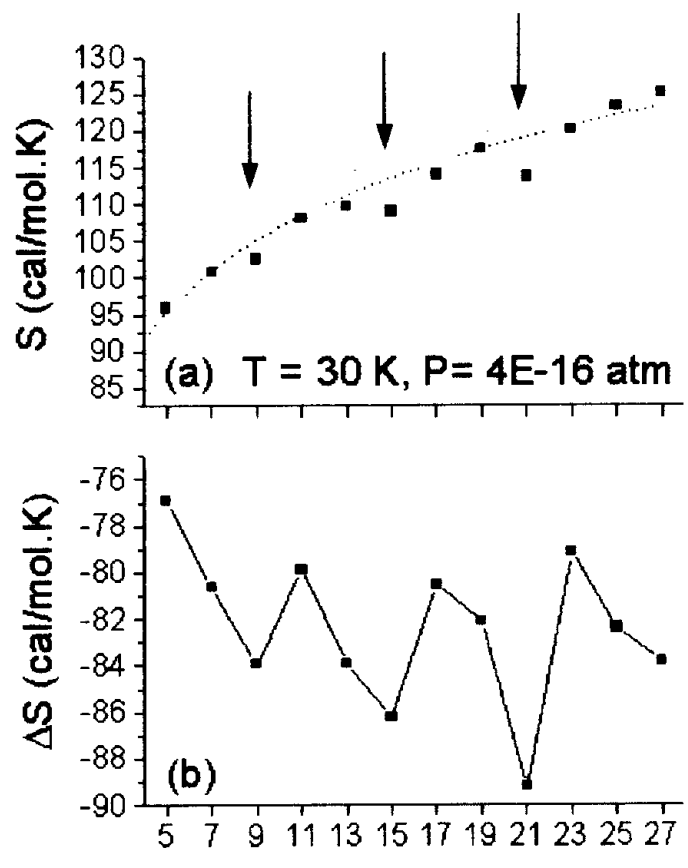

n

Figure 5.(a) Entropy and (b) entropy variation for the $\mathrm{H}_{n}^{+}$cluster (cal/mol.K). The arrows indicate the $n=9,15,21$. The dot curve is a logarithmic fitting.

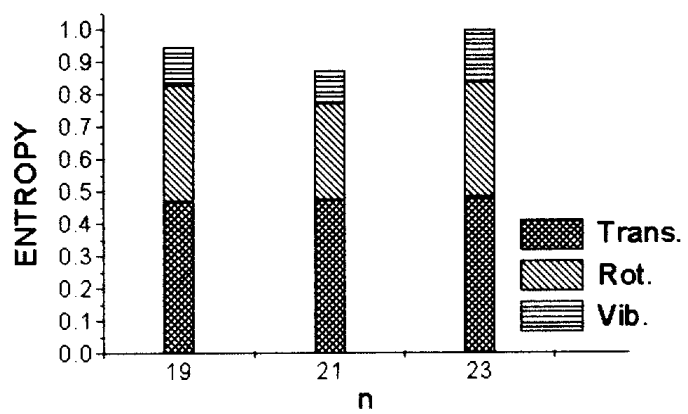

Figure 6. Translational, vibrational and rotational contributions for the entropy of the $n=19,21,23$ clusters. The values are normalized for the total entropy of the $\mathrm{H}_{21}^{+}$.

As shown in Fig.5b, the reduction of the entropy for these three clusters has consequences on the entropy variations, which also present a three-minima pattern. This result is in agreement with the experimental results of Hiraoka [33], who also observed the minima in the entropy variation when the $\mathrm{H}_{9}^{+}, \mathrm{H}_{15}^{+}$and $\mathrm{H}_{21}^{+}$clusters are formed. Particularly in the case of $\mathrm{H}_{15}^{+}$, the minimum seems to indicate that this cluster has predominantly symmetry CtothesamesideoftheH isomer $\left(\mathrm{C}_{S}\right)$, with two $\mathrm{H}_{2}$ molecules at one side and one $\mathrm{H}_{2}$ molecule at the other side of the plane, is non-symmetrical enough to explain the minimum. This result is in agreement with the $a b$ initio calculations [10], which predict that a relatively high rotational barrier should prevent the interconversion between the isomers.

The fact that the minima in the entropy variation are caused by a reduction of the rotational contributions to $\Delta S$ implies that they are not necessarily an indication of closing of a solvation shell. Indeed, according to the results of the ab initio calculations, the $\mathrm{H}_{15}^{+}$and the $\mathrm{H}_{27}^{+}$clusters do not complete their more external occupied shell, but they only have very symmetrical equilibrium geometries.

The Gibbs free energy for Eq.9 depends strongly on the temperature and pressure conditions, as we can see in Fig. 7. For the DIC thermodynamical condition, the result indicates that just the first-shell clusters $(n=5,7,9)$ may be spontaneously produced. For the magnetic trap thermodynamical condition, the result indicates that the first and second-shell clusters $(n=5-21)$ may be spontaneously produced. This result agrees with the experiments of Ref. [44], in which the most abundant cluster observed was the $\mathrm{H}_{19}^{+}$one.

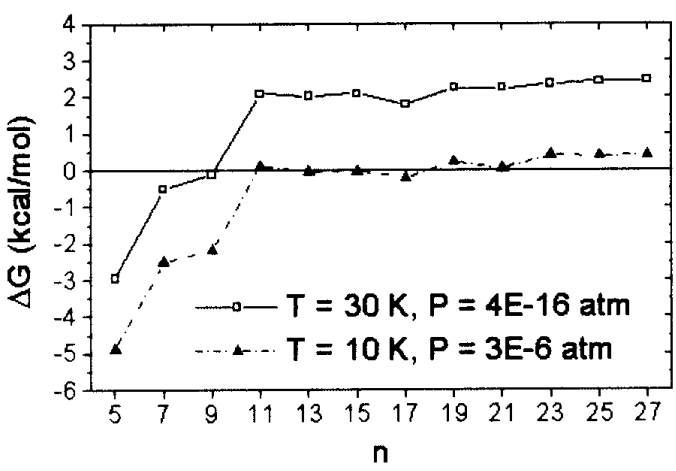

Figure 7. Gibbs free energy variation $(\mathrm{kcal} / \mathrm{mol})$

\section{Conclusions}

We have examined the structural and thermodynamic properties of hydrogen molecular clusters formed around an atomic or molecular cation. For the homogeneous $\mathrm{H}_{n}^{+}$clusters, the shell distribution of $\mathrm{H}_{2}$ molecules shows a pattern in which the first shell has three molecules, the second shell has six molecules, and the third one has about thirteen molecules. Table I presents the number of $\mathrm{H}_{2}$ molecules on the first shell of several inhomogeneous hydrogen clusters.

We have revisited the Bloom-Margenau model for predicting the number of neutral molecules that could bind to a cation core. This classical model works well for high temperatures, when it is used accurate potential energy surfaces. This point is illustrated by taking the $\mathrm{Li}^{+}\left(\mathrm{H}_{2}\right)_{k}$ clusters as a test case.

Finally, we have presented results for the entropy of the $\mathrm{H}_{n}^{+}$clusters ( $n=5-27$, odd) and for the Gibbs free energy variations associated to the cluster formation. The spontaneity of the clustering process, in two different conditions, is examined, and the results have a good agreement with the available experimental data.

\section{Acknowledgments}

The authors would like to thank the support given by the Brazilian agencies CNPq and FAPERJ. 


\section{References}

[1] R. Clampitt and L. Gowland, Nature 223, 815 (1969).

[2] M. Farizon, H. Chermette, and B. Farizon-Mazuy, J. Chem. Phys. 96, 1325 (1992).

[3] D. Scharf, M. L. Klein, and G. J. Martyna, J. Chem. Phys. 97, 3590 (1992).

[4] U. Nagashima, K. Morokuma, and H. Tanaka, J. Phys. Chem. 96, 4294 (1992).

[5] B. Diekmann, P. Borrmann, and E. R. Hilf, Surf. Rev. Lett. 3, 253 (1996)

[6] I. Štich, D. Marx, M. Parrinelo, and K. Terakura, J. Chem. Phys. 107, 9482 (1997).

[7] H. Chermette, H. Razafinjanahary, and L. Carrion, J. Chem. Phys. 107, 10643 (1997).

[8] E.W. Ignacio and S. Yamabe, Chem. Phys. Letters 287, 563 (1998).

[9] B. Farizon, M. Farizon, H. Razafinjanahary, and H. Chermette, Phys. Rev. B 60, 3821 (1999).

[10] M. Barbatti, Ginette Jalbert, and M.A.C. Nascimento, J. Chem. Phys. 113, 4230 (2000).

[11] M. Barbatti, Ginette Jalbert, and M.A.C. Nascimento, J. Chem. Phys. 114, 2213 (2001).

[12] H. Chermette and I.V. Ymmud, Phys. Rev. B 63, 5427 (2001).

[13] H. Müler and W. Kutzelnigg, Phys. Chem. Chem. Phys. 2, 2061 (2000).

[14] R. Prosmiti, A. A. Buchachenko, P. Villareal, and G. Delgado-Barrio, Theor. Chem. Acc. 106, 426 (2001).

[15] M. Barbatti, Ginette Jalbert, and M.A.C. Nascimento, manuscript in preparation (2003).

[16] J. E. Bushnell, P.R. Kemper, and M.T. Bowers, J. Phys. Chem. 98, 2044 (1994).

[17] S. Bloom and H. Margenau, Phys. Rev. 85, 670 (1952).

[18] C.W. Bausclicher, Jr., Harry Partridge, and S.R. Langhoff, J. Phys. Chem. 96, 2475 (1992).

[19] M. F. Falcetta, J.L. Pazun, M.J. Dorko, D. Kitchen, and P.E. Siska, J. Phys. Chem. 97, 1011 (1993).

[20] D. Scharf, G. J. Martyna, and M. L. Klein, J. Chem. Phys., 1993, 99, 8997. D. Scharf, G. J. Martyna, D. Li, G. A. Voth, and M. L. Klein, J. Chem. Phys. 99, 9013 (1993).

[21] R. Davy, E. Skoumbourdis, and T. Kompanchenko, Molec. Phys. 97, 1263 (1999)

[22] M. Barbatti, Ginette Jalbert, and M.A.C. Nascimento, J. Chem. Phys. 114, 7066 (2001).

[23] J. Hinze, O. Friedrich, and A. Sundermann, Mol. Phys. 96, 711 (1999)
[24] P. Weis, P. Kemper, and M. T. Bowers, J. Phys. Chem. A 101, 2809 (1997).

[25] P. R. Kemper, P. Weis, M. T. Bowers, and P. Maître, J. Am. Chem. Soc. 120, 13494 (1998).

[26] P. R. Kemper, J. Bushnell, M. T. Bowers, and G. I. Gellene, J. Phys. Chem. A 102, 8590 (1998).

[27] M. Barbatti, Ginette Jalbert, and M.A.C. Nascimento, J. Phys. Chem. A 106, 551 (2002).

[28] J. Easterfield and J. W. Linnett, Nature 226, 142 (1970).

[29] D. W. Boo and Y. T. Lee, J. Chem. Phys. 103, 520 (1995).

[30] S. Roszak and J. Leszczynski, Chem. Phys. Lett. 323, 278 (2000).

[31] K. Hiraoka, J. Katsuragawa, A. Minamitsu, E. W. Ignacio, and S. Yamabe, J. Phys. Chem. A 102, 1214 (1998).

[32] R.W. Gora, S. Roszak, and J. Leszcynski, J. Phys. Chem. A 103, 9138 (1999)

[33] K. Hiraoka, J. Chem. Phys. 87, 4048 (1987).

[34] N. J. Kirchiner and M. T. Bowers, J. Phys. Chem. 91, 2573 (1987).

[35] B. Farizon, M. Farizon, M. J. Gaillard, and S. Ouaskit, Comput. Materials Sci. 2, 571 (1994).

[36] S. Louc, B. Farizon, M. Farizon, M.J. Gaillard, N. Gonçalves, H. Luna, G. Jalbert, N.V. de Castro Faria, M.C. BacchusMontabonel, J.P. Buchet, and M. Carré, Phys. Rev. A 58, 3802 (1998).

[37] F. Gobet, B. Farizon, M. Farizon, M. J. Gaillard, S. Louc, N. Goncalves, M. Barbatti, H. Luna, Ginette Jalbert, N. V. de Castro Faria, M.C. Bacchus-Montabonel, J.P. Buchet, M. Carré, and T.D. Märk, Phys. Rev. Letters 86, 4263 (2001).

[38] Y.K. Bae, Chem. Phys. Lett. 180, 179 (1991).

[39] V. Špirko, W.P. Kraemer, and P. Soldán, J. Mol. Spectrosc. 183, 218 (1997).

[40] M. Farizon, B. Farizon-Mazuy, N.V. de Castro Faria, and H. Chermette, Chem. Phys. Letters 177, 451 (1991).

[41] Y. Yamaguchi, J.F.Gaw, B. Remington, and H.F.Schaefer III, J. Chem. Phys. 86, 5072 (1987).

[42] M. Okumura, L. I. Yeh, and Y. T. Lee, J. Chem. Phys. 88, 79 (1988).

[43] S. F. Boys and F. Bernardi, Mol. Phys. 1970, 19, 553 (1970).

[44] W. Paul, B. Lücke, S. Schlemmer, and D. Gerlich, Int. J. Mass Spectrom. 149/150, 373 (1995).

[45] B. J. McCall, T. R. Geballe, K. H. Hinkle, and T. Oka, Science 279, 1910 (1998). 